\title{
Clinical course and neurological sequels after tick-borne encephalitis in children - case report
}

\author{
Ilona Palyga-Bysiecka ${ }^{1, A-D \oplus}$, Beata Kręcisz ${ }^{2, E-F} \oplus$, Barbara Szczepańska ${ }^{2, B-C \oplus}$ \\ 1 Jan Kochanowski University, Kielce, Poland \\ ${ }^{2}$ Collegium Medicum, Jan Kochanowski University, Kielce, Poland \\ A - Research concept and design, B - Collection and/or assembly of data, C - Data analysis and interpretation, \\ $D$ - Writing the article, $E$ - Critical revision of the article, $F$ - Final approval of article
}

Palyga-Bysiecka I, Kręcisz B, Szczepańska B. Clinical course and neurological sequels after tick-borne encephalitis in children - case report. Ann Agric Environ Med. 2022; 29(1): 162-167. doi: 10.26444/aaem/133206

\begin{abstract}
Introduction. Tick-borne encephalitis (TBE) is a neurotropic, tick-transmitted infection. Clinical presentation ranges from mild aseptic meningitis to severe encephalitis, and may result in long-term neurological sequels.

Objective. The aim of the study is to present the clinical features of TBE in children, and the laboratory findings, in order to identify neurological sequels and the risk of cognitive deficits in long-term TBE infections.

Materials and method. The study included eight children with serologically-confirmed TBE, hospitalized in the Paediatric Centre in Kielce, Poland.

Results. Despite the more benign clinical course of TBE infections in children compared to adults, the potential for longterm cognitive sequels can be serious. It is hypothesized that a developing CNS is more susceptible to long-term infection effects.

Conclusions. Paediatric TBE should be considered in children with unexplained acute CNS-related symptoms. The longterm consequences may occur with permanent impairment of the quality of life due to neurological consequences.
\end{abstract}

Key words

tick-borne encephalitis, meningitis, cognitive deficits, central nervous system, vaccination

\section{INTRODUCTION}

Tick-borne encephalitis (TBE) is one of the most prelevant human viral disease of the central nervous system caused by the TBE virus (TBEV) belonging to the tick-borne flavivirus group. TBE is an endemic infection which can be found sporadically in populations inhabiting the region stretching from Europe and to the Far East, including China, Siberia and Japan. This localization corresponds to the circulation occurrence of ixodid ticks Ixodes ricinus and Ixodes persualcatus, which are not only the vectors but also a reservoir TBEV. Humans and household animals are accidental tick hosts. The ticks in the endemic areas may contain the virus [1].

\section{OBJECTIVE}

The aim of the study is a revision of the clinical features of tickborne encephalitis in children and the laboratory findings (including CSF), intended to determine the neurological sequels and risk of cognitive consequences in long-term TBE infections.

\section{MATERIALS AND METHOD}

The study utilized the medical reports of 8 children treated between 2006-June 2020 in the Department of Pediatric

Address for correspondence: Ilona Palyga-Bysiecka, Jan Kochanowski University Kielce, Poland.

E-mail: bysiecka@gmail.com

Received: 13.08.2020; accepted: 10.02.2021; first published: 19.03.2021
Infectious and Neurologic Diseases of the Paediatric Centre in Kielce, Poland. In patients with the persisting sequels of the disease at discharge, a life follow-up was conducted for a longer period of time. The diagnosis of tick-borne encephalitis was established using clinical criteria and laboratory confirmation of infection. Diagnosis was confirmed by the enzyme-linked immunosorbent assay (ELISA), by the presence of specific serum IgM and IgG antibodies, or TBEV IgG seroconversion. In established cases of meningoencephalitis, analysis of the cerebrospinal fluid was performed, with an elevated CSF cell count $\left(>5 \times 10^{6}\right.$ cells $\left./ \mathrm{L}\right)$ in the results. Meningitis was confirmed when children presented with headache, irritability, nausea, vomiting, high fever, vertigo with the signs of meningeal irritation with pleocytosis on CS, and serology of TBE. Encephalitis was confirmed when the patient also presented with altered consciousness, somnolence, stupor or coma, tremor of extremities and tongue fasciculations, paroxysmus, cognitive dysfunction, and disturbances in mental activity.

\section{STATE OF KNOWLEDGE}

TBE is an emerging infection, and since 1973 there has been a nearly $400 \%$ growth in all endemic regions of Europe [2]. It has been found in most European countries, except Portugal and Belgium. The prevalence is especially high in Austria, Czech Republic, Poland, Hungary, the Balkan countries and Russia.

The disease is primarily transmitted from late spring until the first months of autumn, when the tick vector is most active, with a peak in July and September in southern Europe, with a longer season in Scandinavia and the Russian Far East. Since the introduction of vaccination against tick- 
borne encephalitis in Austria, there has been a reduction in the incidence of tick-borne encephalitis [3]. Tick-bite is anesthetized and usually unnoticed by the patient. The virus reaches the blood in only a few minutes after the bite. In recent years, the area of TBEV distribution has been spreading, especially in the forest area of north-western Europe. This circumstance is correlated with the global warming causing the migration of TBEV to previously unaffected are, and the increased activity of ticks [4]. Favourable weather conditions in spring and summer, including high temperature and humidity, cause prolonged vegetation and allows both juvenile and adult ticks to look for hosts. Warm weather exhilarates humans to engage in extensive outdoor activities which enhance the risk factors for TBE [5]. The infection is correlated with the transmission through the bite of Ixodes ticks, occasionally by consumption of unpasteurized milk or milk products from infected goat, sheep or cows [1] (Fig. 1). Children in outdoor play grounds and persons while hiking, berry picking or mushroom gathering, may also be at risk, depending on the location and season. The risk of being infected is very high not only for residents of endemic areas, but also for tourists who spend holidays in endemic areas [6].

The clinical course and long-term results depend on the kind of TBEV subtype involved in the infection. Infection with the European TBEV is mostly connected with a biphasic course of the disease, with a mortality rate of $0.5-2 \%$ and severe neurologic course in up to $10 \%$ of patients. In $30 \%$ cases, no tick bite was reported. After a 7-14-day incubation period, the European form is initiated as an acute nonspecific pyretic infection, followed in $5-30 \%$ cases by meningoencephalitis [7]. Of all patients with TBE, meningitis is present in about $50 \%$, meningoencephalitis in about $40 \%$, and meningoencephalomyelitis in about $10 \%$.

The first phase is similar to flu and includes fever, myalgia, weakness and nausea lasting up to one week. At the onset of TBE, the meningeal symptoms are not expressed, and the white cell counts in the cerebrospinal fluid (CSF) are within the normal range. The fever first disappears, but after 2-8 days may reappear, followed by signs and symptoms of CNS inflammation, including nausea, photophobia, and the signs of meningeal irritation in children. Symptoms are presented for 7-10 days with observed improvement after that time. Approximately one-third of those who initially had symptoms will evolve neurological symptoms [8]. The second phase, principally in children, can be characterized by an aseptic meningitis, or it may present as a meningoencephalitis form, poliomyelitic form with flaccid paralysis, or a polyradiculoneuritic form with a Guillain-Barré-like paralysis; all these forms tend to resolve spontaneously. As there is no effective treatment for TBE, prevention is predominant. It is crucial to avoid the exposure of dwellers in habitats that have a high tick density. Although actions promoting TBE prophylaxis and prevention have been undertaken, education about this issue is still incomplete. Personal protection and special safety measures against tick bites, such as proper clothing or use of repellents, have limited efficiency [9], and a skin inspection ('tick check') is not always attainable after exposure [10].

Demographic and epidemiological data - Poland and Świętokrzyskie Region. During the period of this study in Poland, the number of recorded TBE cases varied from 150-250 per year. The regions of particularly substantial morbidity are north-eastern Poland: Podlaskie (41\% of documented cases of TBE, incidence - 9/100,000 people) and the Warmian-Masurian Province (18\% of documented cases TBE, incidence $-3.3 / 100,000$ people) [11]. Travelling to endemic areas in Poland and neighboring countries like the Czech Republic or Slovakia, especially when activity (hiking and camping in forested areas) is planned, represents a highrisk of TBE. As the incubation period of TBE is around 2 weeks, the clinical signs of infections can be observed after returning home. Non-specific influenza-like symptoms of the initial stage of TBE can be correlated with this viral infection by the patients, and usually are not reported to medical care institutions. It is also important to note that the parents and guardians of infants who due to their age are not able to verbalize, may overlook all symptoms of TBE which can therefore remain unrecognized for TBE [12].

Until 2016, the Świętokrzyskie region had been classified as a TBE non-endemic area because prior to that date, only single cases had been diagnosed [11]. In recent years, a

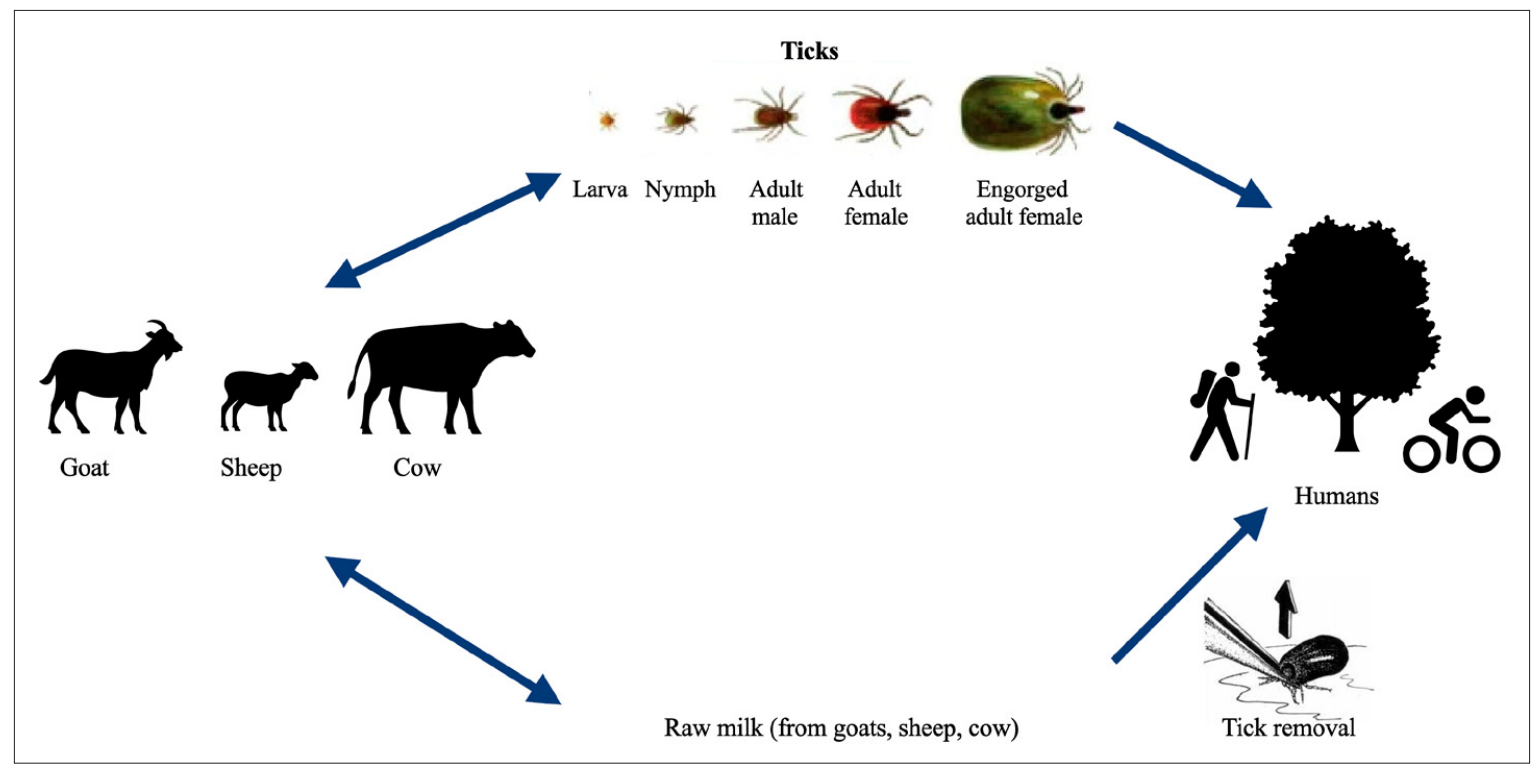

Figure 1. Transmission of tick-borne encephalitis virus 


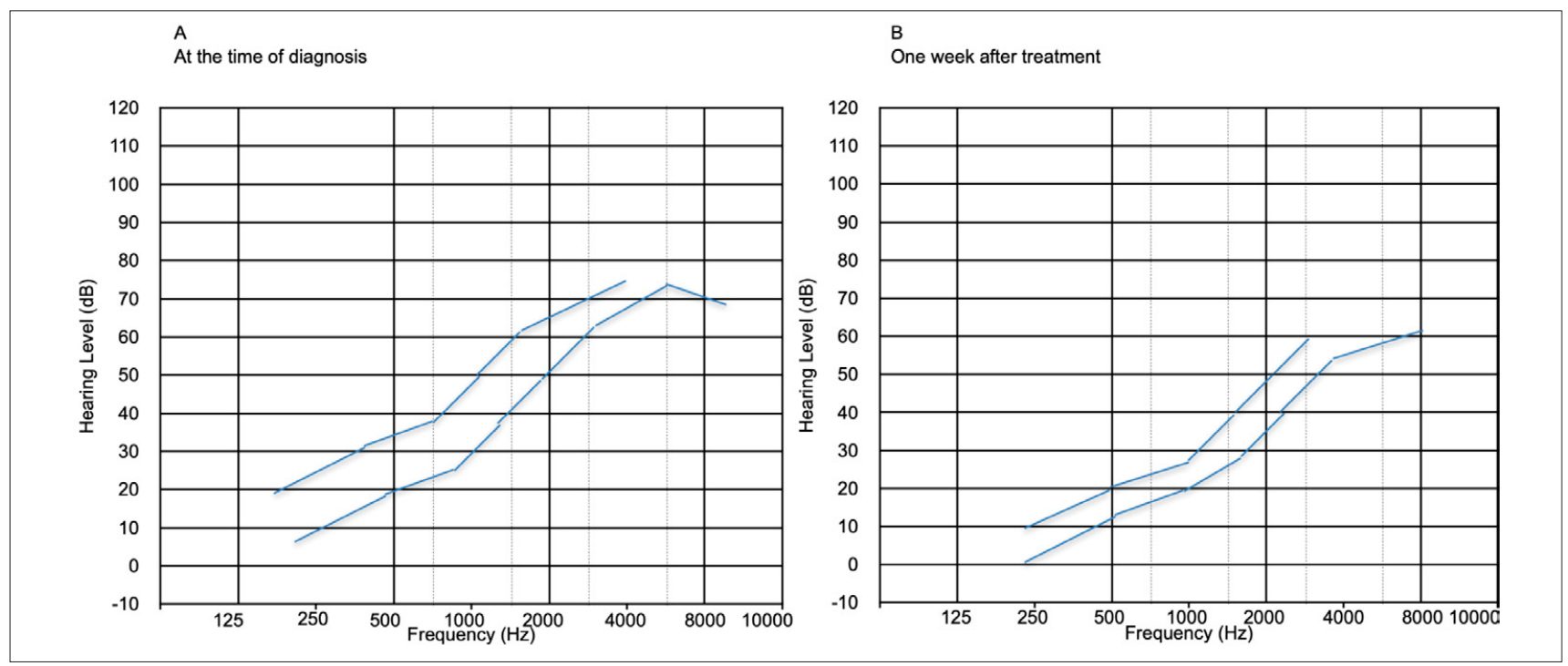

Figure 2. Pure tone audiogram of the left ear

marked increase in the number of TBEV virus infections has been noticed [11]. The disease prevalence in this province is possibly much higher than the number of registered cases due to the plentiful occurrence of I. ricinus ticks which are the vectors and reservoirs of TBEV in this area [13]. In the period 2016- June 2020, 32 patients, of whom 8 were children, have been reported. The Department of Paediatric Infectious and Neurologic Diseases of the Świętokrzyskie Paediatric Centre is the only ward in this region where children with neuroinfections are hospitalized.

TBE infection with the manifestation of central nervous system occurred in 8 patients: 2 had mild, 3 had moderate, and 3 had the severe form of TBE. All TBE cases occurred between April - November with a peak during summertime. In the children with abnormal inflammatory markers in peripheral blood (C-reactive protein, $\mathrm{WBC}$ ), the elevations were only minor. A mildly elevated protein or albumin levels in CSF was seen in 5 patients. Table 1 presents clinical features, epidemiological data, laboratory and CSF findings.

In the presented study, 2 phases of illness was observed in 4 patients. The fever, headache and vomiting during the second stage were noticed in all 8 patients, while meningeal signs were present in 7 children. Meningitis (5 patients) dominated over meningoencephalitis (3 patients). Moreover, the CSF changes caused by inflammation were observed in all 8 children. A mild to moderate pleocytosis with a mononuclear dominance in CSF was observed in all children. Analysis of CSF revealed a normal glucose and lactate level, a moderately raised protein level and pleocytosis in all patients. TBE infection was confirmed by the enzyme-linked immunosorbent assay (ELISA) corroborating TBE-specific IgM and IgG antibodies in the serum. None of the patients had been vaccinated. Severe sequels persisted in 3 children (patient \#1, patient \#5, patient \#8), while in the other two children it was classified as moderate (patient \#2, patient \#4). Meningoencephalomyelitis was not observed. No correlation was found between findings in the cerebrospinal fluid and the severeness of the disease. Computer tomography was without pathologic findings in 6 children.

A half of the patients underwent MRI, among whom one patient had pathological ischaemic lesions in the basal ganglia, and cerebral parenchyma of the left hemisphere. The

Table 1.

\begin{tabular}{|c|c|c|c|c|c|c|c|c|}
\hline Patient no. & 1 & 2 & 3 & 4 & 5 & 6 & 7 & 8 \\
\hline Age of onset & 16 & 17 & 14 & 15 & 14 & 15 & 16 & 17 \\
\hline Tick-bite & yes & no & no & no & no & no & no & yes \\
\hline Biphase course & no & yes & yes & no & yes & no & no & yes \\
\hline Fever & 5 & 0 & 4 & 6 & 4 & 4 & 7 & 3 \\
\hline Meningeal sign & yes & no & yes & yes & yes & yes & yes & yes \\
\hline Pareses & no & yes & no & no & no & no & no & no \\
\hline Strabismus, nystagmus & no & no & no & no & yes & no & no & no \\
\hline Hyperkinesia & no & no & yes & no & yes & no & no & no \\
\hline CSF cells $(\mu \mathrm{g} / \mathrm{L})$ & 688 & 109 & 889 & 270 & 1044 & 280 & 128 & 41 \\
\hline CSF Protein (g/L) & 0.54 & 0.95 & 0.62 & 0.5 & 1.03 & 0.55 & 0.9 & 0.68 \\
\hline IgM Serum (NTU) & 5 & 7.8 & 6.11 & 5.55 & 3.22 & 4.67 & 34.38 & 48 \\
\hline IgG Serum (NTU) & 49.79 & 160.64 & 115.19 & 119.23 & 150.5 & 139.4 & 27.12 & 21 \\
\hline
\end{tabular}


patient (patient \#2), a 17-year-old girl, was admitted to the clinic with pareses and significant involuntary trembling of the left upper limb. Since a secondary hypoactivity of thyroid gland was reported in laboratory findings, she was given replacement therapy). Her new brain MRI was normal. The first child (patient \#1), who was diagnosed with encephalitis, presented with somnolence, quantitative and qualitative disturbances of consciousness with drowsiness and confusion, and periodic excitement and aggression. During hospitalization, patient did not remember the place of his residence and facts from his life. He was expressing delusions based on disturbances of perception. Another patient (\#5) presented with oedema of the brain with bradycardia and altered consciousness. He was admitted to hospital with cerebral dysfunction, strabismus and dizziness.

The final patient, a 17-year-old male (\#8), was admitted to hospital with sudden onset of psychomotor excitement with altered of consciousness, auditory and visual hallucinations (he saw insects on his skin). The use of force was necessary because of his aggression during first days of hospitalization, and he was consulted by psychiatrist. The day before admission, he had also presented with illogical aggression, alternated with laughing and crying.

Because of hearing difficulties during hospitalization, he was consulted by laryngologist and underwent impendance audiometry which confirmed sensoneurinal hearing loss on the left side. In two children (patient \#1 and \#4), concomitant neuroboreliosis was determined by positive intrathecal synthesis of IgM/IgG antibodies against Borrelia burgdorferrii. They were both treated with IIIrd generation cefalosporine (ceftriaxone) for 21 days, with no allergy reactions after treatment. These two children were residents of the same region, living within a two kilometer radius of one another. One year later, a girl (patient \#1) had recurring Lyme disease which presented as local knee arthritis, with an elevated level of antibodies IgM and IgG against Borrelia burgdorferi. The clinical picture in both patients with coexisting TBE and neuroborreliosis had significant severe and moderate consequences. In patient \#3 and patients \#6-7, the sequels were classified as mild with minor impact on the quality of life. All patient received symptomatic treatment in the form of analgesics and antipyretics and had control of electrolyte and fluid balance; if necessary, anticonvulsive agents were used. Disabled limbs needed physiotherapy to prevent muscular atrophy. All patients completely recovered and returned to their daily activities.

\section{DISCUSSION}

Tick-borne encephalitis, following tick bites, is the most frequent febrile illness in European endemic areas, with about $15 \%$ of all registered cases of TBE represented by subjects aged $<19$ years [13]. Infection mainly affects young patients with a median age of nine years, the youngest patient with TBE infection were 17 days-old (Austria), 6 weeks-old (Switzerland), 3 months-old (one each in Austria and Czech Republic and 4.5 months-old (Germany) children [14]. Nonspecific symptoms - malaise and fever - explain why children are not correctly diagnosed. In particular, TBE should be considered in any unclarified acute CNS-related symptoms in young children residing in endemic regions, and a TBErelated serological investigation should be performed [15].
TBE should be suspected in every child with meningitis, encephalitis or myelitis in a TBEV endemic area, because the diagnosis is most effective within the incubation period for TBE. Individuals with severe encephalitis should first be treated with acyclovir ( $10 \mathrm{mg} / \mathrm{kg}$ three times daily), until herpes simplex virus encephalitis is excluded [16]. The two patients (patient \#1, patient \#5) in the current study were treated by intravenous acyclovir until the diagnosis of TBE was established.

In the endemic regions, approximately $10 \%$ of the childhood causes of encephalitis are incited by TBEV [8]. While the clinical symptoms of TBE are usually milder in children, the illness may also have more severe courses. Several reports have shown [17] that the long-term outcomes from TBE infection in childhood can be unfavourable due to cognitive problems linked with disturbed brain activity. Persistent neurological sequels are defined if the symptoms persisted six month after discharge from hospital. In the current study, neurocognitive deviations, including lasting tremor of the upper extremities, speech impairment, wakefulness, atypical hyperkinetic movements and irritability, were found in four hospitalized children (patients \#2, \#3, \#5 and \#8). The cognitive abnormalities were detected for executive function and working memory.

The difficulties in recognizing neurodevelopmental deficits as a result of childhood TBE may have supported the widespread perception and common understanding of TBE as a mild ailment of the juvenile period. The persistent signs of the disease described by parents were fatigue, headache and irritability; memory perception difficulties were also observed.

TBE is often described as a mild disease [7], but this has been challenged recently [18]. Schmolck et al. [18] have suggested that young patients experiencing TBE performed poorly in the psychometric tests of executive function, compared with controls. They also exhibited behavioural abnormalities, school problems and concentration deficits which occurred in up to $25 \%$ of the patients. Engman et al. [19], who monitored eight TBE-infected children for a year, demonstrated that long-term morbidities (drowsiness, irritability) were commonly seen in TBE patients, and based on their observations, suggested that TBE was not a mild infection in children.

In the current study, behavioural disorders were observed in two children (patient \#1 and \#5) who had impaired concentration, and were described by their parents as having problems with initiating activities and working memory. One girl who underwent TBE 2.5 year ago, at follow-up still has significant involuntary trembling of the distal parts of limbs at rest. Another boy (patient\# 2), three years later, was still experiencing trembling of the upper extremities while lifting. A recent long-term follow-up study in 55 children [20] has shown that $75 \%$ of children experience residual problems, mainly associated with cognitive disorders, headache, fatigue and irritability. However, serious consequences (e.g. grave neurological disability or death) were infrequently seen in paediatric TBE.

The presented study revealed that a high percentage of children treated for tick-borne encephalitis who had central nervous system involvement, continued to have symptoms after two to seven years, with the most prevalent cognitive problems in executive function and working memory. Neuropsychiatric disorders, such as attention and 
concentration deficits, were persistent in $2 \%$ of paediatric patients [20]. There is no convincing prognostic marker in the acute phase to predict long-term outcome; mild TBE was not indicative of a better prognosis $[18,20]$. Two of the patients in the current study (\#4 and patient\#5), experienced memory problems during the follow-up period; a similar phenomenon was described by Engman et al. [19]. There was no connection between the age at illness onset and acuity severity of symptoms at follow-up, suggesting that even a young patient might suffer from an incomplete recovery, manifesting later as they grew up. The symptoms of the disease were so marked that they required educational support during school time and daily life.

The female patient (patient \#2) with secondary hypoactivity of thyroid gland that started after the diagnosis of TBE, received replacement treatment which is ongoing, and for the last two years until now is under the observation of an endocrinologist. Prior to the TBE, she had never had a history of thyroid gland illness. No report has been in the literature on the correlation between TBE and secondary hypoactivity of thyroid gland.

Patient \#8 had a unilateral sensorineural hearing loss confirmed on tonal and impedance audiometry, he also had extended high-frequency tonal audiometry (up to 18 $\mathrm{kHz}$ ) and speech audiometry tests. The first adult patient with sensoneurinal deafness associated with TBE has been reported in England [21]. Another study [22] confirmed sensorineural hearing loss in $16.7 \%$ of patients treated for tick-borne diseases, mainly Lyme disease, or being coinfected by TBE. There is no way to distinguish how many patients had been infected with TBE. A systemic literature search showed no relevant articles revealing a correlation between TBE and sensorineural hearing loss in children. The correlation between both secondary hypoactivity of thyroid gland and sensorineural hearing loss may be associated with a history of TBE encephalitis.

After meningitis, the signs of cognitive dysfunction may be unmarked when the follow-up is not conducted. Increased awareness of delayed cognitive deficits following TBE infection in children with noticeable attention and memory disturbances, can initiate a prompt treatment by stimulants aided with computer games, as employed for children with ADHD. The most frequent TBEV-linked neurological deficits may lead to disturbances in cognitive and motor function which often cause serious long-term disabilities. Following encephalitis, a lasting disability was also observed which may result from residual focal cerebral lesions or a postencephalitic state with cognitive and psychiatric symptoms [7, 8]. Mild-to-moderate neurologic sequels or cognitive deficits affecting the child's learning and everyday activities have been described. Many signs of mental disorders, including impaired consciousness, amnesia, mental deterioration, behavioural changes, psychosis and restlessness, may also appear. Patients also develop such minor neuropsychological deficits as persistent attention and concentration deficits. Since TBE has a prolonged impact on the fate of children and their families, it is important to protect the child from cognitive impairment [23].

The most prevalent manifestations described for the postencephalitic syndrome include several cognitive complaints (i.e. learning difficulties, reduced stress tolerance), defective learning skills, headache, movement coordination disorder, dysphasia and hearing defects [7]. Frequent kinds of neurological sequels are cranial nerve paresis, hemiparesis or paraparesis, shoulder amyotrophy, and impaired coordination and balance [24].

Although brain and spinal cord imaging exhibit both a low sensitivity and low specificity, nevertheless the technique is useful for differential diagnosis. CT of the brain is regarded as less sensitive than MRI for the diagnosis of encephalitis, but it is advised in an emergency situation before lumbar puncture when brain oedema is conjectured, or for differential diagnosis [25]. Magnetic resonance imaging showed nonspecific TBE-associated parenchymal lesions in $20-33 \%$ of all patients [26]. Typical TBE changes include hypodense regions and has a bizarre predilection for basal ganglia and thalamus localization, the multifocal or diffuse lesions can be distributed unilaterally or bilaterally [26]. The follow-up of MRI investigation of patients with serious TBE proved that corticosubcortical atrophy with augmented ventricles was more conspicuous on the follow-up examination, compared to the imaging at admission [25]. A half of the patients in the current study underwent MRI, of which one patient had pathological ischemic injuries in the basal ganglia and brain parenchyma of the left hemisphere; he also had a pareses and significant involuntary trembling of the left upper limb.

The crucial consequence of post-encephalitic syndrome is that children residing in or arriving at an endemic region should be given a vaccination against TBE $[27,28,29]$, which is the only effective protection against TBEV before exposure to ticks, since there is no advice for passive and active immunization following a tick-bite. The overall vaccination rate in Poland is $0.5 \%$ [30], compared to Czech Republic where it is 19\% [31]. The most distinctive effect was found in Austria, where $95 \%$ of children are vaccinated [28].

Infections in the developing CNS have to be taken seriously. The presented cases contribute additional evidence that tickborne encephalitis in young patients has a considerable morbidity, and in single individuals, serious long-lasting neurological sequels can occur.

\section{CONCLUSIONS}

Childhood TBE CNS infections should be more frequently recognized, and serologic investigation paying attention to TBE taken into consideration, especially when travel to an endemic region was involved. An atypical course of infection without initial fever phase or meningeal signs should also be anticipated. Larger prospective studies will provide better understanding of the prevalence and longterm sequels of childhood tick-borne CNS infections. The cognitive impairment after TBE in children is more common than formerly thought. The possible CNS infections following tick bites call for a need for lifelong follow-up, and first of all, the application of available preventive measures. A substantial morbidity of TBE observed in children highlights the importance of vaccination. Possible life-long effects, such as cognitive dysfunction, remain to be investigated.

\section{REFERENCES}

1. Haditsch M. Ticks and tick-borne encephalitis in Europe: Challenges for travel medicine. Travel Med Infect Dis. 2018; 26: 5-6. https:/doi. org/10.1016/j.tmaid.2018.11.010 
2. Estrada-Peña A, Gray JS. Research on the ecology of ticks and tick-borne pathogens--methodological principles and caveats. Front Cell Infect Microbiol. 2013; (8)3: 29. https://doi.org/10.3389/fcimb.2013.00029

3. Qviller L, Grøva L, Viljugrein H, Klingen J, Mysterud A. Temporal pattern of questing tick Ixodes ricinus density at differing elevations in the coastal region of western Norway. Parasit Vectors. 2014; 7: 179 https://doi.org/10.1186/1756-3305-7-179

4. Haemig PD, Sjostedt de Luna S, Grafstrom A, et al. Forecasting risk of tick-borne encephalitis (TBE): using data from wildlife and climate to predict next year's number of human victims. Scand J Infect Dis. 2011; 43: 366-372. https://doi.org/10.3109/00365548.2011.552072

5. Steffen R. Epidemiology of tick-borne encephalitis (TBE) in international travellers to Western/Central Europe and conclusions on vaccination recommendations. J Travel Med. 2016; 23: 4. https://doi. org/10.1093/jtm/taw018

6. Dobler G, Gniel D, Petermann R, Pfeffer M. Epidemiology and distribution of tick-borne encephalitis. Wien Med Wochenschr. 2012; 162: 230-238. https://doi.org/10.1007/s10354-012-0100-5

7. Kaiser R. Tick-borne encephalitis. Nervenarzt. 2016; 87(6): 667-680. https://doi.org/10.1007/s00115-016-0134-9

8. Barp N, Trentini. Clinical and laboratory findings in tick-borne encephalitis virus infection. Parasite Epidemiol Control. 2020; 19: 10: e00160. https://doi.org/10.1016/j.parepi.2020.e00160

9. Bogovic P, Strle F. Tick-borne encephalitis: a review of epidemiology, clinical characteristics, and management. World J Clin Cases. 2015; (3)5: 430-441. https://doi.org/10.12998/wjcc.v3.i5.430

10. Buchel K, Bendin J. Repellent efficacy of DEET, Icaridin, and EBAAP against Ixodes ricinus and Ixodes scapularis nymphs. Ticks Tick Borne Dis. 2015; 6(4): 494-498. https://doi.org/10.1016/j.ttbdis.2015.03.019

11. Narodowy Instytut Zdrowia Publicznego, Meldunki epidemiologiczne. Choroby zakaźne i zatrucia w Polsce w roku 2019r. http://wwwold.pzh. gov.pl/oldpage/epimeld/2019/Ch_2019_Wstepne_dane.pdf.

12. Bartosik K, Lachowska-Kotowska P. Environmental conditioning of incidence of tick-borne encephalitis in the south-eastern Poland in 1996-2006. Ann Agric Environ Med. 2011; 18(1): 119-126.

13. ECDC. Tick-borne encephalitis. Annual Epidemiological Report for 2018. https://www.ecdc.europa.eu/en/publications-data/tick-borneencephalitis-annual-epidemiological-report-2018

14. Steffen R. Tick-borne encephalitis (TBE) in children in Europe. Ticks and Tick-borne Diseases. 2019; 10(1): 100-110. https://doi.org/10.1016/j. ttbdis.2018.08.003

15. Sundin M. Pediatric tick-borne infections of the central nervous system in an endemic region of Sweden: a prospective evaluation of clinical manifestations Eur J Pediatr. 2012; 171(2): 347-352. https://doi. org/10.1007 s00431-011-1542-2

16. Gaieski DF, Nathan BR, O’Brien NF. Emergency neurologic life support: meningitis and encephalitis. Neurocrit Care. 2015; 12(23): S110-S118. https://doi.org/10.1007/s12028-015-0165-2
17. Ruzek D, et al. Tick-borne Encephalitis in Europe and Russia: Review of Pathogenesis, Clinical Features, Therapy and Vaccines. Antiviral Res. 2019; 164: 23-51. https://doi.org/10.1016/j.antiviral.2019.01.014

18. Schmolck H, Maritz E, Kletzin I, Korinthenberg R. Neurologic, neuropsychologic, and electroencephalographic findings after European TBE in children. J Child Neurol. 2005; 20(6): 500-508. https://doi. org/10.1177/088307380502000606

19. Engman ML, Lindstrom K, Sallamba M, Hertz C, Sundberg B, Hansson $\mathrm{ME}$, et al. One-year follow-up of tick-borne central nervous system infections in childhood. Pediatr Infect Dis J. 2012; 31: 570-574. https:// doi.org/10.1097/inf.0b013e31824f23c0

20. Fowler A, Forsman L, Eriksson M, Wickström R. Tick-borne encephalitis carries a high risk of incomplete recovery in children. J Pediatr. 2013; 163, 555-560. https://doi.org/10.1016/j.jpeds.2013.01.037

21. McNair A, Brown L. Tick-borne encephalitis complicated by monoplegia and sensorineural deafness. J Infect. 1991; 22: 81-86.

22. Sowula K, Składzie J. Otolaryngological symptoms in patients treated for tick-borne diseases Otolaryngol Pol. 2018 Feb; 72(1): 30-34. https:// doi.org/10.5604/01.3001.0011.5948

23. Sundin M. TBE in children.The Book. Global Health Press, Singapore. 2017. pp. 85-90.

24. Taba P, Schmutzhard E, Forsberg P. EAN consensus review on prevention, diagnosis and management of tick-borne encephalitis. Eur J Neurol. 2017; 24(10): 1214-e61. https://doi.org/10.1111/ene.13356

25. Czupryna P, Tarasow E, Moniuszko-Malinowska A, et al. MRI and planimetric CT follow-up study of patients with severe tick-borne encephalitis. Infect Dis. 2016; 48: 1-8.

26. Zawadzki R, et al. Evaluation of imaging methods in tick-borne encephalitis. Pol J Radiol. 2017; 82: 742-747. https://doi.org/10.12659/ pjr.903940

27. Beck R. Fritz K. Molecular basis of the divergent immunogenicity of two pediatric tick-borne encephalitis virus vaccines. J Virol. 2016; 90: 4.1964-1972. https://dx.doi.org/10.1128\%2FJVI.02985-15

28. Zavadska D, Anca I. Recommendations for tick-borne encephalitis vaccination from the Central European Vaccination Awareness Group (CEVAG). Hum Vaccin Immunother. 2013; 9(2): 362-374. https://doi. org/10.4161/hv.22766.Epub2013Jan4

29. Galgani I, Bunge E. Systematic literature review comparing rapid 3-dose administration of the GSK tick-borne encephalitis vaccine with other primary immunization schedules. Expert Rev Vaccines. 2017; 16: 919-932. https://doi.org/10.1080/14760584.2017.1358620

30. Szczepienia ochronne w Polsce w 2018 roku. Biuletyn Narodowego Instytutu Zdrowia Publicznego-Państwowego Zakładu Higieny. http:// wwwold.pzh.gov.pl/oldpage/epimeld/2018/Sz_2018.pdf.

31. Erber W, Schmitt HJ. Self-reported tick-borne encephalitis (TBE) vaccination coverage in Europe: Results from a cross-sectional study. Ticks Tick Borne Dis. 2018; 9(4): 768-777. https://doi.org/10.1016/j. ttbdis.2018.02.007 